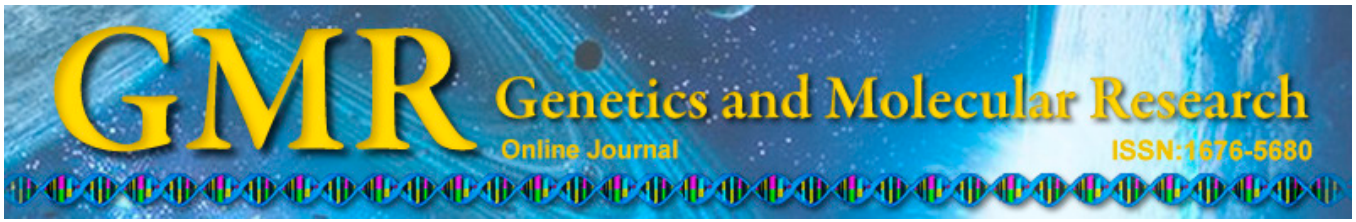

\title{
Protective effects of morphine preconditioning in delayed phase on myocardial ischemia- reperfusion injury in rabbits
}

\author{
X.-H. Lu' ${ }^{1}$ K. Ran', Y.-Y. Xiao' ${ }^{1}$, D.-L. Yang', Y.-T. Chang', K.-M. Duan² \\ and Y.-W. $\mathrm{Ou}^{2}$ \\ ${ }^{1}$ Department of Anesthesiology, the Second Xiangya Hospital, \\ Central South University, Changsha, China \\ ${ }^{2}$ Department of Anesthesiology, the Third Xiangya Hospital, \\ Central South University, Changsha, China \\ Corresponding author: K. Ran \\ E-mail: yangdonglin_ydl@yeah.net
}

Genet. Mol. Res. 14 (3): 8947-8954 (2015)

Received June 5, 2014

Accepted February 10, 2015

Published August 7, 2015

DOI http://dx.doi.org/10.4238/2015.August.7.3

\begin{abstract}
The aim of this study was to investigate the protective mechanisms of delayed-phase morphine preconditioning on myocardial ischemia-reperfusion injury. Thirty healthy male New Zealand white rabbits were randomly divided into three groups: a sham operation group (C), ischemia-reperfusion group (I/R), and delayed-phase morphine preconditioning group (M) $(\mathrm{N}=10$ /group). Rabbits in the $\mathrm{C}$ group received thoracotomy for $160 \mathrm{~min}$. Rabbits in the $\mathrm{I} / \mathrm{R}$ group received left artery blockage for $40 \mathrm{~min}$ and reperfusion for $120 \mathrm{~min}$. Rabbits in the $\mathrm{M}$ group received $1.0 \mathrm{mg} / \mathrm{kg}$ intravenous morphine $24 \mathrm{~h}$ prior to the identical treatment as the rabbits in the I/R group. In each group, the interleukin (IL)-10 and tumor necrosis factor (TNF)- $\alpha$ levels were detected at five time points: 20 min before the left coronary artery blockage (T1), 20 and 40 min after the left coronary artery blockage (T2 and T3, respectively), and 1 and $2 \mathrm{~h}$ after the myocardial reperfusion (T4 and T5, respectively). After reperfusion, the infarction size was
\end{abstract}


measured with Evans blue and 2,3,5-triphenyltetrazolium chloride (TTC) staining. Compared with the C group, serum IL-10 and TNF- $\alpha$ concentrations increased in the $\mathrm{I} / \mathrm{R}$ and $\mathrm{M}$ groups; the difference was significant $(\mathrm{P}<0.05)$. When compared with the I/R group, the IL-10 concentrations in the $M$ group were significantly increased $(P<0.05)$, but the infarction size and TNF- $\alpha$ concentrations were significantly decreased $(\mathrm{P}<0.05)$. These results suggested that delayed-phase morphine preconditioning might achieve myocardial protection through the regulation and balance of inflammatory cytokines.

Key words: Morphine; Preconditioning in delayed phase; Cytokines; Myocardial protection; Ischemia-reperfusion injury

\section{INTRODUCTION}

Ischemia-reperfusion (I/R) injury is an urgent clinical problem that needs to be solved. Studies have shown that preconditioning has a protective effect on myocardial I/R injury. The preconditioning protective effect could be divided into two stages: as an early or delayed phase, according to the time provided (Bolli, 1996). Delayed-phase preconditioning (DPC) is characterized by a prolonged genetic response for the second time and an enhancement of new protein synthesis, which is triggered $12-24 \mathrm{~h}$ after the reaction. The advanced protection is expected to be maintained for a duration of 2-3 days. It has been affirmed that morphine preconditioning demonstrated a level of myocardial protection similar to that observed for early-phase ischemic preconditioning (Groban et al., 2004; Okubo et al., 2004). However, it remains unknown whether morphine preconditioning also has a delayed myocardial protective effect. Interleukin (IL)-10 (Giomarelli et al., 2003) and tumor necrosis factor (TNF)- $\alpha$ (Liu et al., 2006a) are the inflammatory cytokines that play the most important roles in myocardial $\mathrm{I} / \mathrm{R}$ pathological damage. Therefore, in this study we observed the effects of morphine preconditioning on IL-10 and TNF- $\alpha$ levels in I/R injured rabbits to explore the mechanism of morphine preconditioning on delayed myocardial protection.

\section{MATERIAL AND METHODS}

\section{Materials}

\section{Animals}

The study comprised 30 healthy adult male New Zealand white rabbits weighing 2.0$2.5 \mathrm{~kg}$; all animals were provided by the Animal Department of Xiangya Medical College. All animals were fed and processed in accordance with the Hunan Provincial Medical Experimental Animal Feeding and Management Regulations and International Laboratory Animal Care and Management Regulations.

\section{Equipment}

The equipment used in this study and their sources were: DH150 ventilator (Medical 
Instrument Factory of Zhejiang Medical University); MD100-2 electronic analytical balance (Shanghai Balance Instrument Factory, Shanghai, China); automatic biochemical analyzer (Beckman Coulter, Brea, CA, USA); endo-type homogenizer machine (Zhongshan Medical College Instrument Factory); 5415R high-speed low-temperature tabletop centrifuge (Eppendorf, Hamburg, Germany); 721 spectral photometer (Shanghai Third Analytical Instrument Factory); constant temperature water bath (Shanghai Instrument Factory); $-70^{\circ} \mathrm{C}$ refrigerator (Panasonic Corp., Osaka, Japan); pressure transducer (Hewlitt Packard Company); 450-nm microplate reader, Japanese 550 type BIO-RAD Model (Bio-Rad Laboratories, Berkeley, CA, USA).

\section{Reagents}

Reagents used in this study included Evans Blue, Nitro Blue Tetrazolium Chloride (2,3,5-triphenyltetrazolium chloride; TTC), sodium pentobarbital, and acrylamide (Sigma-Aldrich, St. Louis, MO, USA). IL-10 and TNF- $\alpha$ enzyme-linked immunosorbent assay (ELISA) kits were purchased from Jingmei, Beijing, China.

\section{Methods}

\section{I/R model preparation}

To model I/R in rabbits, $30 \mathrm{mg} / \mathrm{kg}$ sodium pentobarbital was injected into the ear veins as an anesthetic. After the animals were immobilized, tracheotomy and intubation were performed for mechanical ventilation using pure oxygen, with a tidal volume of $15 \mathrm{~mL} / \mathrm{kg}$ and a respiratory rate of 35 breaths $/ \mathrm{min}$. The thoracotomy was performed on the 4 th intercostal space of the left anterior wall. The pericardium was cut open, and the middle part of the left coronary artery was blocked with silk ligature to cause the blood flow blockage and myocardial ischemia. As confirmation, the ischemic ventricular wall showed cyanosis and bulging accompanied with ST-segment elevation in the electrocardiogram.

\section{Animal grouping}

The study groups consisted of a total of 30 rabbits, which were randomly divided into three groups: the sham operation group $(\mathrm{C})$, ischemia-reperfusion group (I/R), and the morphine pretreatment group (M), each containing 10 rabbits. Rabbits in the $\mathrm{C}$ group received $1.0 \mathrm{~mL} / \mathrm{kg}$ intravenous normal saline; after $24 \mathrm{~h}$, the left coronary artery was lined without blocking for $160 \mathrm{~min}$. Rabbits in the I/R group received $1.0 \mathrm{~mL} / \mathrm{kg}$ intravenous NS; after $24 \mathrm{~h}$, the left descending artery was blocked for $40 \mathrm{~min}$ and reperfused for $120 \mathrm{~min}$. Rabbits in the $\mathrm{M}$ group received $1.0 \mathrm{mg} / \mathrm{kg}$ intravenous morphine; after $24 \mathrm{~h}$, the treatment was the same as for the rabbits in the $\mathrm{I} / \mathrm{R}$ group.

\section{Detection of study indicators}

\section{Determination of IL-10 and TNF- $\alpha$ protein expression levels}

In each group, the IL-10 and TNF- $\alpha$ levels in the internal carotid arterial blood were detected by sandwich ELISA at the following five time points: $20 \mathrm{~min}$ before occlusion of the 
left descending coronary artery (T1), 20 and $40 \mathrm{~min}$ after the left descending coronary artery occlusion (T2 and T3, respectively), and 1 and $2 \mathrm{~h}$ after the myocardial reperfusion (T4 and $\mathrm{T} 5$, respectively).

\section{Measurement of myocardial infarction size}

After $2 \mathrm{~h}$ of reperfusion, the coronary artery was blocked again, and $2 \mathrm{~mL} \mathrm{2 \%} \mathrm{Evans}$ blue was injected in the internal carotid artery reversely to stain the myocardial tissue to differentiate ischemic (non-blue) and non-ischemic (blue) tissue. The hearts were removed quickly and cut horizontally into approximately $2-\mathrm{mm}$ slices, dried with filter papers, and the right ventricle and the non-ischemic tissue were cut off and measured for quality determination. The ischemic myocardium slices were incubated with $0.5 \%$ TTC phosphate buffer $(\mathrm{pH}=$ 7.4) at $37^{\circ} \mathrm{C}$ for $15 \mathrm{~min}$ to determine the infarction tissues. The necrotic (gray-white) and non-necrotic (dark red) areas were separated and measured for quality, and the ischemia and infarction myocardial size was calculated as a percentage of the total left ventricular mass.

\section{Examination of myocardial ultrastructure}

After reperfusion, 1- $\mathrm{mm}^{3}$ apex myocardial tissues were taken in each group for ultrastructural examination under transmission electron microscopy.

\section{Statistical analysis}

All data are reported as means \pm standard deviation, and analysis of variance was performed with the SPSS 13.0 statistical software (SPSS, Chicago, IL, USA). P $<0.05$ was taken as a significant statistical difference.

\section{RESULTS}

\section{Serum concentrations of IL-10 and TNF- $\alpha$}

Compared with the $\mathrm{C}$ group, serum IL-10 concentrations were significantly increased in the I/R and $\mathrm{M}$ groups after I/R $(\mathrm{P}<0.05)$. However, compared with the I/R group, the IL-10 concentrations in the $\mathrm{M}$ group were significantly increased $(\mathrm{P}<0.05)$. Compared with the $\mathrm{C}$ group, serum TNF- $\alpha$ concentrations were significantly increased in the I/R and $\mathrm{M}$ groups after $\mathrm{I} / \mathrm{R}(\mathrm{P}<0.05)$, but compared with the $\mathrm{I} / \mathrm{R}$ group, the TNF- $\alpha$ concentrations in the M group were significantly decreased $(\mathrm{P}<0.05)$, as shown in Table 1 .

Table 1. Serum IL-10 and TNF- $\alpha$ concentrations in each group (means \pm SD).
\begin{tabular}{llccccc}
\hline Indicators & Group & T1 & T2 & T3 & T4 & T5 \\
\hline IL-10 $(\mathrm{pg} / \mathrm{mL})$ & $\mathrm{C}$ & $3.1 \pm 1.0$ & $2.7 \pm 1.5$ & $3.2 \pm 0.8$ & $3.3 \pm 1.2$ & $2.9 \pm 1.8$ \\
& $\mathrm{I} / \mathrm{R}$ & $3.4 \pm 1.4$ & $5.6 \pm 1.8$ & $7.5 \pm 4.1^{*}$ & $9.5 \pm 3.6^{*}$ & $12.1 \pm 3.1^{*}$ \\
& $\mathrm{M}$ & $3.2 \pm 1.8$ & $5.2 \pm 1.4$ & $11.6 \pm 3.4^{*}$ & $15.4 \pm 4.1^{* *}$ & $19.3 \pm 2.7^{* *}$ \\
$\mathrm{TNF}-\alpha(\mathrm{pg} / \mathrm{mL})$ & $\mathrm{C}$ & $52.3 \pm 6.7$ & $54.2 \pm 4.5$ & $53.7 \pm 3.1$ & $54.6 \pm 4.2$ & $51.5 \pm 5.6$ \\
& $\mathrm{I} / \mathrm{R}$ & $54.1 \pm 5.3$ & $78.6 \pm 6.4$ & $88.1 \pm 7.9^{*}$ & $110.5 \pm 9.4^{*}$ & $157.6 \pm 5.5^{*}$ \\
& $\mathrm{M}$ & $56.7 \pm 7.4$ & $61.3 \pm 5.6$ & $72.6 \pm 5.4^{*}$ & $91.8 \pm 6.9^{* *}$ & $113.8 \pm 8.2^{* *}$ \\
\hline
\end{tabular}

*Compared with the $\mathrm{C}$ group, $\mathrm{P}<0.05$. ${ }^{\sharp}$ Compared with the $\mathrm{I} / \mathrm{R}$ group, $\mathrm{P}<0.05$. 


\section{Myocardial infarction size}

There was no significant difference in the ischemic size between the I/R and $M$ groups $(\mathrm{P}>0.05)$. Compared with the $\mathrm{I} / \mathrm{R}$ group, infarction size in the $\mathrm{M}$ group was significantly smaller $(\mathrm{P}<0.05)$ (Table 2).

Table 2. Myocardial infarction size and ischemic area (means $\pm \mathrm{SD})$.

\begin{tabular}{lccccc}
\hline Group & LV $(\mathrm{g})$ & AAR $(\mathrm{g})$ & IS $(\mathrm{g})$ & AAR/LV $(\%)$ & IS/AAR $(\%)$ \\
\hline $\mathrm{C}$ & $3.16 \pm 0.68$ & - & - & - & - \\
$\mathrm{I} / \mathrm{R}$ & $2.93 \pm 0.44$ & $0.88 \pm 0.35$ & $0.33 \pm 0.06$ & $33.6 \pm 3.1$ & $37.8 \pm 1.7$ \\
$\mathrm{M}$ & $3.07 \pm 0.56$ & $1.09 \pm 0.29$ & $0.24 \pm 0.05$ & $35.4 \pm 2.6^{*}$ & $21.5 \pm 2.4^{*}$ \\
\hline *Compared with the IR group, P $>0.05 ;{ }^{*}$ Compared with the I/R group, $\mathrm{P}<0.05 . \mathrm{LV}=$ left ventricular mass; AAR \\
$=$
\end{tabular}

\section{Myocardial ultrastructure changes}

Under the electron microscope, the myocardial mitochondria in the sham operation group $\mathrm{C}$ showed a normal morphology and abundant glycogen. In the $\mathrm{I} / \mathrm{R}$ group, myocardial mitochondria showed edema, severe vacuolization, and fuzzy boundaries. The myocardial mitochondria in the morphine group M showed mild swelling and reduced glycogen (Figures 1-3).



Figure 1. Morphological changes of myocardial cells in the C group (20,000X). 


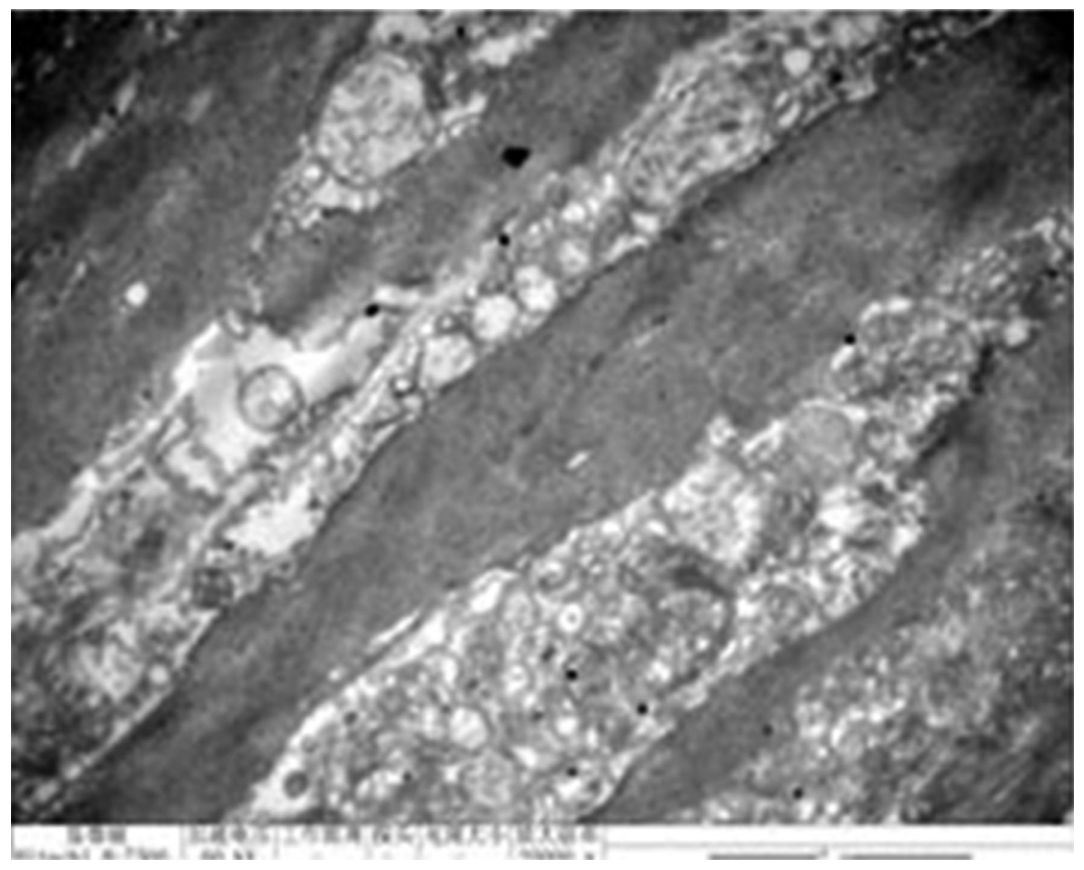

Figure 2. Morphological changes of myocardial cells inthe I/R group (20,000X).

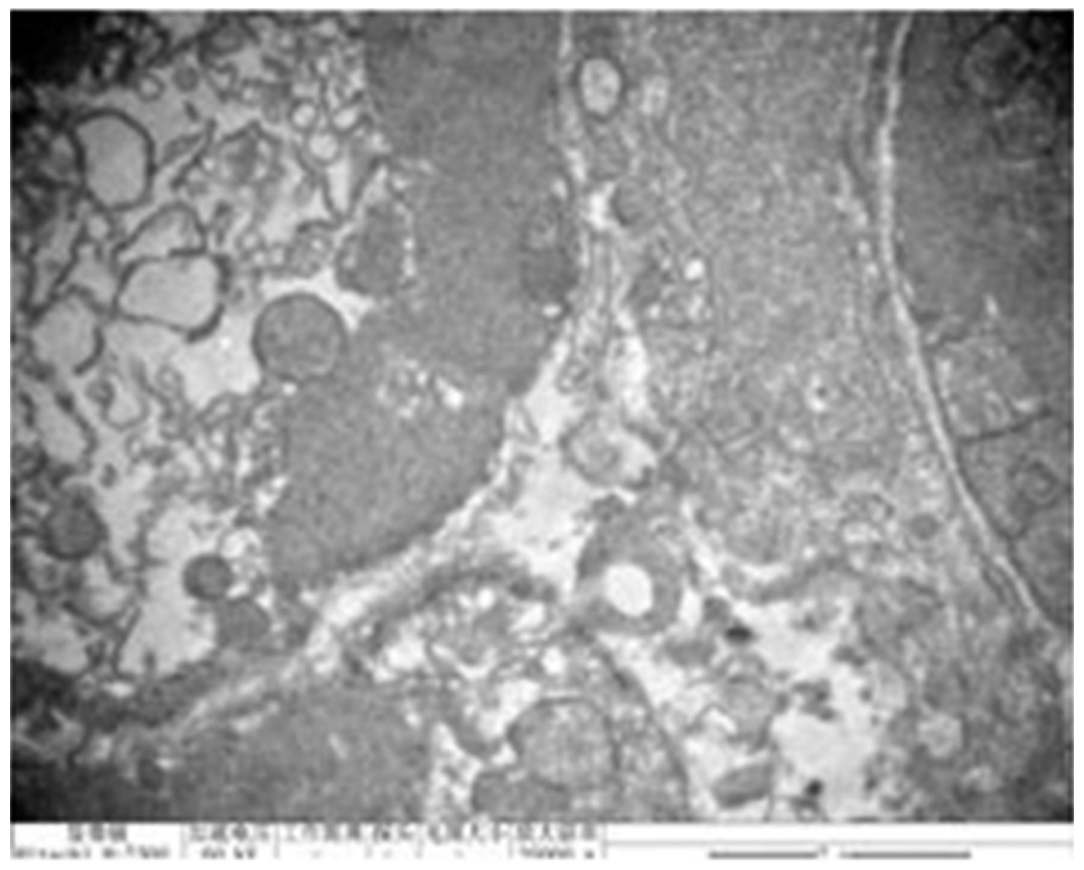

Figure 3. Morphological changes of myocardial cells in the M group (20,000X). 


\section{DISCUSSION}

One of the mechanisms of I/R is the excessive inflammatory response. TNF- $\alpha$ is the major pro-inflammatory factor involved in the inflammatory response in the body. It is the critical medium in the chain reaction of myocardial I/R injury; rapidly generated, it can cause a cascade of cytokine production resulting in promotion of the inflammatory response (Sack et al., 2002). During I/R, TNF- $\alpha$ release increases, which was shown to result in activated polymorphonuclear leukocytes (PMN), increased aggregation and expression of intercellular adhesion molecule-1 (ICAM-1) and vascular cell adhesion molecule (VCAM-1) in the ischemic area, and promotion of neutrophil accumulation and the release of proteolytic enzymes, which together might cause cell membrane damage, cell autolysis, and, eventually, myocardial injury (Frangogiannis et al., 2002). In the present study, delayed-phase morphine preconditioning significantly reduced serum TNF- $\alpha$ production and infarction size, suggesting that delayed phase morphine preconditioning may exert myocardial protection effects through the inhibition of TNF- $\alpha$ production and relief of inflammation at the site of $\mathrm{I} / \mathrm{R}$ injury.

IL-10 is a classic anti-inflammatory cytokine that can suppress the synthesis of many proinflammatory factors such as TNF- $\alpha$, IL-1, and IL-6, and elevate production of other antiinflammatory cytokines (Howard and O'Garra, 1992). Several studies have found that IL-10 has an important role in the protection of myocardial ischemic injury, and that expression of $I L-10$ mRNA and protein is significantly upregulated during I/R (Frangogiannis et al., 2000). If IL-10 expression was not present, the inflammatory response was amplified, which was expressed as enhanced PMN infiltration, increased ICAM- 1 and TNF- $\alpha$ aggregation and expression, expanded area of myocardial infarction, and more severe overall damage (Yang et al., 2000). In the present study, after morphine DPC, myocardial IL-10 expression and production significantly increased, suggesting that the I/R injury protective effects observed following morphine DPC may result from the upregulation of IL-10 production and inhibition of the inflammatory reaction.

Myocardial ultrastructure change is an effective indicator of the degree of myocardium injury (Ohtake et al., 2004). In the present study, electron microscoscopic examination showed markedly swollen myocardial cells, interstitial edema, vacuolar degeneration of the cytoplasm, disordered arrangement of myofilaments, high levels of edema and vacuolar degeneration in the sarcoplasm and mitochondria, as well as obvious interstitial edema in the I/R group. After $24 \mathrm{~h}$ of DPC, the microscopic examination showed only a slight swelling of myocardial cells, mild interstitial edema with rare vacuolar degeneration, clear lines of myofilaments, no obvious interstitial edema, and sarcoplasmic and mitochondrial swelling without vacuolar degeneration in the M group. These results suggested that morphine DPC could effectively reduce the degree of myocardial injury, and this was associated with inhibition of excessive inflammatory response.

Myocardial infarction size change is considered to be the gold standard in the evaluation of myocardial preconditioning effects (Zaugg et al., 2003). In the present study, the ischemic area showed no significant difference between the I/R and M groups, indicating the certainty of the left anterior descending artery locking effect, and that the LAD occlusion sites were consistent across different groups. The infarction size was $37.8 \%$ in the I/R group, while it was only $19.7 \%$ in the morphine DPC M group $24 \mathrm{~h}$ after the preconditioning. Compared with the I/R group, infarction size in the $\mathrm{M}$ group was reduced by $48 \%$. These results suggested that morphine DPC might reduce infarction size in I/R injury $24 \mathrm{~h}$ after preconditioning. The 
effect was closely related to the inhibition of I/R-induced inflammatory reaction. Previous studies (Yeh et al., 2005; Liu et al., 2006b) have shown that I/R induced the overexpression of TNF- $\alpha$, IL-1, IL-6, and other proinflammatory cytokines through activation of nuclear factor (NF)-kappaB protein expression to cause the inflammatory response of myocardial injury. The downregulation of NF-kappaB protein expression could inhibit the over expression of TNF- $\alpha$, IL-1, IL-6, and other proinflammatory cytokines and raise the expression of IL-10 to eventually reduce the infarction size. Further studies are needed to confirm whether the morphine preconditioning might downregulate the expression of NF-kappaB and inhibit the inflammatory response to reduce the myocardial injury.

In conclusion, this study showed that morphine DPC might inhibit the inflammatory response in the pathological processes of $\mathrm{I} / \mathrm{R}$ through downregulation of the production of proinflammatory TNF- $\alpha$ and upregulation of the generation of the anti-inflammatory cytokine IL-10. This may reflect one of the mechanisms of the protective effects of morphine DPC on myocardial I/R injury.

\section{ACKNOWLEDGMENTS}

Research supported by the Science and Technology Bureau projects of Changsha, Hunan (\#K1003049-31) and a Hunan Provincial Health Department Project (\#B2011-029).

\section{REFERENCES}

Bolli R (1996). The early and late phases of preconditioning against myocardial stunning and the essential role of oxyradicals in the late phase: an overview. Basic Res. Cardiol. 91: 57-63.

Frangogiannis NG, Mendoza LH, Lindsey ML, Ballantyne CM, et al. (2000). IL-10 is induced in the reperfused myocardium and may modulate the reaction to injury. J. Immunol. 165: 2798-2808.

Frangogiannis NG, Smith CW and Entman ML (2002). The inflammatory response in myocardial infarction. Cardiovasc. Res. 53: 31-47.

Giomarelli P, Scolletta S, Borrelli E and Biagioli B (2003). Myocardial and lung injury after cardiopulmonary bypass: role of interleukin(IL)-10. Ann. Thorac. Surg. 76: 117-123.

Groban L, Vernon JC and Butterworth J (2004). Intrathecal morphine reduces infarct size in a rat model of ischemiareperfusion injury. Anesth. Analg. 98: 903-909.

Howard M and O'Garra A (1992). Biological properties of interleukin 10. Immunol. Today 13: 198-200.

Liu X, Xie W, Liu P, Duan M, et al. (2006a). Mechanism of the cardioprotection of rhEPO pretreatment on suppressing the inflammatory response in ischemia-reperfusion. Life Sci. 78: 2255-2264.

Liu X, Shen J, Jin Y, Duan M, et al. (2006b). Recombinant human erythropoietin (rhEPO) preconditioning on nuclear factor-kappa B (NF-kB) activation \& proinflammatory cytokines induced by myocardial ischaemia-reperfusion. Indian J. Med. Res. 124: 343-354.

Ohtake M, Morino S, Kaidoh T and Inoué T (2004). Three-dimensional structural changes in cerebral microvessels after transient focal cerebral ischemia in rats: scanning electron microscopic study of corrosion casts. Neuropathology 24: 219-227.

Okubo S, Tanabe Y, Takeda K, Kitayama M, et al. (2004). Ischemic preconditioning and morphine attenuate myocardial apoptosis and infarction after ischemia-reperfusion in rabbits: role of delta-opioid receptor. Am. J. Physiol. Heart Circ. Physiol. 287: H1786-H1791.

Sack MN, Smith RM and Opie LH (2002). Tumor necrosis factor in myocardial hypertrophy and ischaemia-an antiapoptotic perspective. Cardiovasc. Res. 45: 688-695.

Yang Z, Zingarelli B and Szabo C (2000). Crucial role of endogenous interleukin-10 production in myocardial ischemia/ reperfusion injury. Circulation 101: 1019-1026.

Yeh CH, Chen TP and Wu YC (2005). Inhibition of NFkappaB activation with curcumin attenuates plasma inflammatory cytokines surge and cardiomyocytic apoptosis following cardiac ischemia/reperfusion. J. Surg. Res. 125: 109-116.

Zaugg M, Lucchinetti E, Garcia C, Pasch T, et al. (2003). Anaesthetics and cardiac preconditioning. Part II. Clinical implications. Br. J. Anaesth. 91: 566-576. 